\title{
Angiotensin II and aldosterone-induced neuronal damage in neurons through an astrocyte-dependent mechanism
}

\author{
Li-Juan Min, Masaki Mogi, Jun Iwanami, Akiko Sakata, Fei Jing, Kana Tsukuda, Kousei Ohshima and \\ Masatsugu Horiuchi
}

The contribution of the renin-angiotensin-aldosterone system (RAAS) to central nervous system (CNS) disorders is not yet fully understood. RAAS has been shown to be involved in the proliferation of astrocytes, which have a role in neuronal damage contributing to neurodegenerative diseases. However, the direct relationship between RAAS and neuronal damage is still unclear. We therefore examined the effect of angiotensin (Ang) II and aldosterone (Aldo) on damage to spinal ganglion neurons (SGNs) by regulating astrocytes. Ang II stimulation significantly increased DNA damage in SGNs in a time-dependent manner. This increase in DNA damage was further enhanced when SGNs were co-cultured with astrocytes. On the other hand, no significant increase was observed in SGNs co-cultured with astrocytes without Ang II stimulation. Moreover, the addition of conditioned medium from Ang II-treated astrocytes exacerbated SGN DNA damage. An Ang II type 1 receptor blocker, valsartan, inhibited Ang II-stimulated DNA damage but not DNA damage induced by conditioned medium prepared from astrocyte cultures. In contrast, an Aldo antagonist, eplerenone, significantly inhibited DNA damage induced by the culture medium from Ang II-treated astrocytes. Ang II-stimulated Aldo secretion in the conditioned medium from astrocytes. Furthermore, the administration of Aldo alone also enhanced DNA damage in SGNs. Finally, flow cytometric analysis showed that Ang II or Aldo treatment markedly increased the percentage of dead SGNs. In conclusion, Ang II- and Aldo-induced neuronal damage in SGNs through astrocytes regulation. Blocking Ang II and Aldo to target astrocytes might be useful for the treatment of CNS disorders. Hypertension Research (2011) 34, 773-778; doi:10.1038/hr.2011.38; published online 7 April 2011

Keywords: aldosterone; angiotensin II; astrocyte; central nervous system disorder; neuronal damage

\section{INTRODUCTION}

Central nervous system (CNS) disorders, including neurodegenerative diseases such as Alzheimer disease, Parkinson disease and amyotrophic lateral sclerosis (ALS), are characterized by neuronal dysfunction and degeneration within various regions of the brain and/or the spinal cord. ${ }^{1}$ Astrocytes are the most numerous non-neuronal cell type in the CNS, and they perform multiple functions essential to normal neuronal modulation. ${ }^{2}$ However, astrocytes have recently been implicated in the dynamic regulation of neuronal degeneration in several specific neurodegenerative diseases. It has been suggested that the astrocyte-induced increase in neuronal C1q expression could damage neuronal integrity and cause the loss of neurons, leading to the pathogenesis of Alzheimer disease. ${ }^{3}$ More recent studies revealed that astrocytes expressing a mutated form of the gene for the ubiquitously expressed $\mathrm{Cu} / \mathrm{Zn}$ superoxide dismutase (SOD) 1 protein induce neuronal death in ALS patients. ${ }^{4}$ These findings indicate that astrocytes might participate in neurotoxic mechanisms to induce neuronal damage, leading to CNS dysfunction. There are reports further suggesting that astrocytes might be associated with the induction of oxidative stress to promote DNA damage resulting in neuronal degeneration. ${ }^{5,6}$ However, the more detailed cellular mechanisms involved in the neuron-astrocyte interaction that regulates neuronal damage remain largely unknown.

The renin-angiotensin-aldosterone system (RAAS) is well known to contribute to hypertension and cardiovascular disease, and inhibitors of RAAS are widely used as antihypertensive and cardiovasculature-protective drugs. ${ }^{7}$ However, accumulating evidence shows that RAAS that is localized in the CNS also has an important role in CNS disorders. ${ }^{8,9}$ Recently, increased attention has focused on understanding the involvement of RAAS in the pathogenesis of neurodegenerative diseases. Kawajiri et al. ${ }^{10}$ observed a reduced Ang II level in the cerebrospinal fluid of patients with ALS. Moreover, in vitro and in vivo studies showed that an inhibitor of RAAS, olmesartan, has a neurotrophic effect on neurons in the spinal cord of the ALS rat, 
implicating a potential therapeutic use of olmesartan in inhibiting neuronal degeneration for the treatment of neurodegenerative diseases. ${ }^{11}$ However, the direct role of RAAS in regulating neuronal damage is not clear.

Angiotensin (Ang) II and aldosterone (Aldo) are two important effectors of RAAS and have a variety of pathophysiological actions via binding to the Ang II type $1\left(\mathrm{AT}_{1}\right)$ receptor and mineralocorticoid receptor (MR), respectively. ${ }^{12,13}$ Along with others, we have demonstrated that Ang II often cross-talks with Aldo to mediate cardiovascular remodeling through an interaction between the $\mathrm{AT}_{1}$ receptor and MR activation. ${ }^{14,15}$ The $\mathrm{AT}_{1}$ receptor and MR have been clearly identified in numerous specific regions in the brain and spinal cord. ${ }^{16,17}$ Moreover, it has been suggested the $\mathrm{AT}_{1}$ receptor and $\mathrm{MR}$ are expressed in cultured astrocytes and neurons ${ }^{18,19}$ and mediate Ang II-induced astrocyte proliferation. ${ }^{18}$ Numerous studies indicate that Ang II and Aldo are key stimulators of oxidative stress, which can trigger multiple signaling mechanisms in the CNS and in the cardiovascular system..$^{20,21}$ Therefore, we speculate that the interaction of Ang II with Aldo might be related to neuronal damage with possible involvement of astrocyte function through oxidative stress, thereby contributing to the pathogenesis of CNS disorders. To address this hypothesis, we examined the effects of Ang II and Aldo on neuronal damage by evaluating a marker of oxidative DNA damage using cultured spinal ganglion neurons (SGNs). We also employed SGN-astrocyte co-cultures to clarify the involvement of astrocytes in Ang II- and Aldo-induced damage to neurons.

\section{METHODS}

\section{Cell culture}

This study was performed in accordance with the National Institutes of Health guidelines for the use of C57BL/6J mice (Clea Japan, Osaka, Japan). Neurons were prepared from embryonic day 20 mouse spinal ganglia between the thoracic and sacral level, as described previously, with minor modifications according to the standard culture technique of nerve tissue. ${ }^{22}$ After dissection, SGNs were dissociated by incubation with $2.5 \%$ trypsin (Invitrogen, Carlsbad, CA, USA) and DNase for $30 \mathrm{~min}$ at $37^{\circ} \mathrm{C}$. SGNs were then mechanically triturated into a single-cell suspension with a pipette and syringe in Dulbecco's modified Eagle's medium (Life Technologies, Gaithersburg, MD, USA) supplemented with $10 \%$ fetal bovine serum (Invitrogen), $100 \mathrm{ng} \mathrm{ml}^{-1}$ nerve growth factor (Sigma-Aldrich, St Louis, MO, USA) and antibiotics (PenStrep; Invitrogen). After centrifugation and resuspension in growth medium, the cells were plated in six-well dishes prepared for co-culture with astrocytes and cultured at $37^{\circ} \mathrm{C}$ in a humidified atmosphere of $5 \% \mathrm{CO}_{2}$ and $95 \%$ air. Three days after plating, cultures were treated with $10^{-6} \mathrm{moll}^{-1}$ cytosine arabinoside for 2 days, yielding a stable population of neurons without supporting Schwann cells or fibroblasts. The neurons were then maintained in fresh growth medium that was replaced every 2 days for 1 week until use. The purity of cultured SGNs was $>95 \%$, as detected by the immunofluorescent staining of microtubuleassociated protein 2 (MAP2).

Astrocytes were obtained from neonatal day 0-2 mice, as described previously, with minor modifications according to the standard culture technique. ${ }^{23}$ Briefly, newborn mice were decapitated and the cerebral cortices were removed. After removal of the meninges, the cerebral cortices were digested with $0.4 \mathrm{mg} \mathrm{ml}^{-1}$ dispase (Invitrogen) for $15 \mathrm{~min}$. The digested tissue was dissociated with a pipette and syringe in Dulbecco's modified Eagle's medium supplemented with $10 \%$ fetal bovine serum and antibiotics and passed through a strainer (Biosciences Discovery Labware, Two Oak Park, Bedford, MA, USA) to remove organized debris. The cells were cultured in 6-well or 24 -well dishes at $37{ }^{\circ} \mathrm{C}$ in a humidified atmosphere of $5 \% \mathrm{CO}_{2}$ and $95 \%$ air. Twenty-four hours after the initial plating, the medium was changed to preserve adhering astrocytes and to remove neurons and oligodendrocytes. The cultures were maintained in growth medium that was replaced every 2 days for 1 week until the experiments. The purity of cultured astrocytes was $>95 \%$, as detected by the immunofluorescent staining of glial fibrillary acidic protein (GFAP).
For SGN-astrocyte co-cultures, astrocytes were seeded in six-well dishes with a Falcon Cell Culture Insert (FCCI) of $1-\mu \mathrm{m}$ pore size (Becton Dickinson Labware, Franklin Lakes, NJ, USA). After stimulation with or without Ang II for $24 \mathrm{~h}$, astrocytes with a FCCI were placed on the top of the six-well dishes cultured with SGNs, and the co-cultures were maintained in SGN culture medium for the indicated times.

\section{Quantification of 8-hydroxy-2'-deoxyguanosine (8-OHdG)}

The immunoreactivity of 8-OHdG in the DNA of SGN was detected by an enzyme-linked immunosorbent assay. ${ }^{24}$ Briefly, genomic DNA was extracted using a Wizard Genomic DNA Purification Kit (Promega, Madison, WI, USA), and the 8-OHdG level in $20 \mu \mathrm{g}$ sample DNA was measured using an 8-OHdG Check ELISA Kit (Japan Institute for the Control of Aging, Shizuoka, Japan). The ratio of ng $8-\mathrm{OHdG} \mathrm{m}^{-1}$ DNA was calculated.

\section{Measurement of Aldo concentration}

The Aldo concentration was measured as previously described. ${ }^{14}$ Subconfluent and quiescent cells that were cultured in 24-well dishes in $1 \mathrm{ml}$ Dulbecco's modified Eagle's medium growth medium per well were incubated with or without Ang II in phenol red-free Dulbecco's modified Eagle's medium for the indicated times. At the end of the incubation period, a 200- $\mu \mathrm{l}$ aliquot of the supernatant was removed to measure the Aldo concentration using an Aldo ELISA Kit (Alpha Diagnostic International, San Antonio, TX, USA). The cells were isolated with trypsin to determine the cell count.

\section{Cell death detection assay}

SGN death was determined by flow cytometric analysis using a BD Cell Viability Kit (BD Biosciences, San Jose, CA, USA) according to the manufacturer's instructions. Briefly, cells were harvested and resuspended in staining buffer consisting of phosphate-buffered saline, $0.01 \%$ Tween- 20 and $1 \mathrm{mmoll}^{-1}$ EDTA. Five minutes after staining with thiazole orange solution and propidium iodide at room temperature, the cells were analyzed using a flow cytometer (FACSCalibur, BD, Franklin Lakes, NJ, USA) in combination with software (CellQuest, BD).

\section{Materials}

Valsartan was donated by Novartis Pharma AG (Basel, Switzerland), and Eplerenone was donated from Pfizer (New York, NY, USA). All other reagents not mentioned above were purchased from Sigma-Aldrich.

\section{Statistical analysis}

All values in the text and figures are expressed as mean \pm s.e.m. Data were evaluated by analysis of variance followed by post hoc analysis for multiple comparisons. Differences with $P<0.05$ were considered significant.

\section{RESULTS}

The presence of astrocytes enhances Ang II-induced DNA damage in SGNs

We examined the effect of Ang II on DNA damage in SGNs via the possible cross-talk with astrocytes by quantitating 8-OHdG levels in an SGN-astrocytes co-culture system. Cell cultures were divided into five groups: (1) SGN only, (2) SGN stimulated with Ang II directly, (3) SGN with FCCI with Ang II addition for $24 \mathrm{~h}$, (4) SGN with FCCI cultured with astrocytes and (5) SGN with FCCI cultured with Ang II-treated astrocytes for $24 \mathrm{~h}$. These culture systems were each maintained for 1, 3 or 5 days, and 8-OHdG levels in the SGNs were then measured. Ang II stimulation ( $100 \mathrm{~nm}$ ) significantly increased 8-OHdG levels in SGNs in a time-dependent manner by direct (group 2) and indirect (group 3) stimulation methods. These 8-OHdG levels in SGNs were further enhanced when the cells were co-cultured with Ang II-stimulated astrocytes (group 5). However, co-culture with astrocytes alone did not increase the 8-OHdG levels in SGNs (group 4) (Figure 1). These results suggest that Ang II might promote SGN DNA damage via cross-talk with astrocytes. 


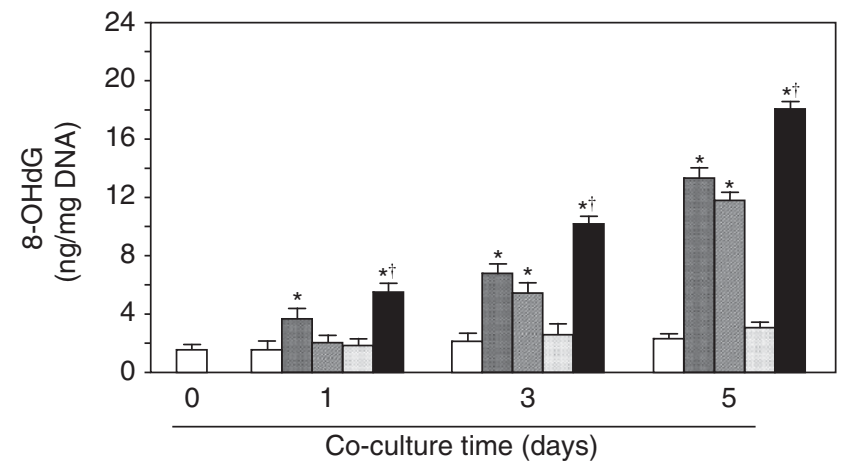

Figure 1 Enhancement of DNA damage in spinal ganglion neurons (SGNs) by angiotensin (Ang) II via the possible cross-talk with astrocytes. Subconfluent and quiescent SGNs were stimulated for $24 \mathrm{~h}$ with or without Ang II directly or with an inserted Falcon Cell Culture Insert (FCCI) with Ang II or co-cultured for $24 \mathrm{~h}$ with astrocytes alone or with Ang II-treated astrocytes; these culture systems were maintained for 1,3 or 5 days. Levels of 8-hydroxy-2'-deoxyguanosine (8-OHdG) in the SGN DNA were determined by enzyme-linked immunosorbent assay (ELISA), as described in Methods section. Values are expressed as mean \pm s.e.m. $(n=4)$. $\square$, SGN only; SGN stimulated with Ang II directly; $\mathbb{Z}$, SGN with FCCI with Ang II addition for $24 \mathrm{~h}$; 3 , SGN with $\mathrm{FCCl}$ cultured with astrocytes; $\square$, SGN with $\mathrm{FCCl}$ cultured with Ang II-treated astrocytes for $24 \mathrm{~h}$. Ang II, angiotensin II (100 nm). ${ }^{*} P<0.05$ vs. SGN only at 0 day. ${ }^{\dagger} P<0.01$ vs. SGN stimulated with Ang II directly and SGN with FCCI with Ang II addition at 1, 3, 5 days, respectively.

Eplerenone, but not valsartan, inhibits DNA damage in SGNs cultured in Ang II-treated astrocyte-conditioned medium

Next, we examined the effect of an $\mathrm{AT}_{1}$ receptor blocker, valsartan, on Ang II-induced SGN DNA damage in relation to astrocytes. Cultures were designed to subject SGNs to different treatments for 3 days as follows: without any stimulation; stimulated with Ang II in the presence or absence of valsartan; stimulated with culture medium of Ang II with or without valsartan-treated astrocytes; stimulated with Ang II and culture medium from Ang II-treated astrocytes (conditioned medium); stimulated with valsartan or eplerenone and conditioned medium. SGNs were subjected to different treatments for 3 days by changing the old medium every $24 \mathrm{~h}$ with freshly prepared medium containing Ang II and/or valsartan and freshly prepared astrocytes culture medium. Astrocytes were stimulated with Ang II with or without valsartan for $24 \mathrm{~h}$. Ang II or conditioned medium increased 8-OHdG levels in SGNs, and 8-OHdG levels in SGNs stimulated with Ang II and conditioned medium were higher than those in SGNs stimulated with Ang II or conditioned medium alone. The administration of valsartan $(10 \mu \mathrm{M})$ significantly inhibited the Ang II-induced 8-OHdG levels in SGNs. However, valsartan did not alter the 8-OHdG levels in SGNs stimulated with the conditioned medium (Figure 2). In contrast, a mineralocorticoid receptor antagonist, eplerenone $(10 \mu \mathrm{M})$, strongly blocked 8 -OHdG levels in SGNs stimulated with the conditioned medium regardless of the presence of valsartan (Figure 3). These results show that Aldo secreted from Ang II-stimulated astrocytes might have an important role in inducing DNA damage in SGNs.

\section{Aldo secreted by Ang II-stimulated astrocytes promotes DNA damage in SGNs}

To examine whether Aldo is secreted by Ang II-simulated astrocytes, astrocytes were stimulated with or without Ang II by changing the old medium every $24 \mathrm{~h}$ with freshly prepared medium with or without

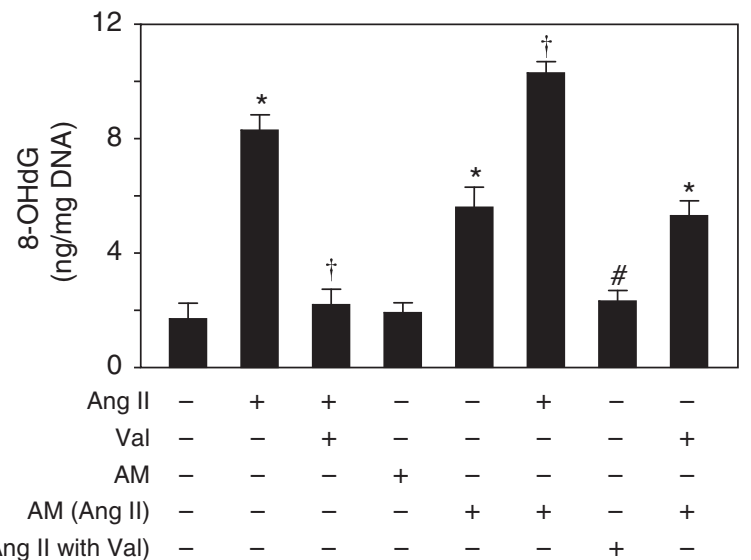

Figure 2 Valsartan inhibited Ang II-induced DNA damage in spinal ganglion neurons (SGNs), but not Ang II-treated astrocyte culture medium-induced SGN DNA damage. Subconfluent and quiescent SGNs were stimulated with or without Ang II in the presence or absence of valsartan, and/or stimulated with culture medium from Ang II with or without valsartan-treated astrocytes, or stimulated with valsartan or eplerenone and culture medium from Ang II-treated astrocytes. SGNs were stimulated for 3 days and then subjected to 8-hydroxy-2'-deoxyguanosine (8-OHdG) quantification. Values are expressed as mean \pm s.e.m. $(n=4)$. Ang II, angiotensin II $(100 \mathrm{~nm})$; Val, valsartan $(10 \mu \mathrm{M})$; AM, astrocyte medium; AM (Ang II), Ang II-treated astrocyte medium; AM (Ang II with Val), Ang II with valsartan-treated astrocyte medium. ${ }^{*} P<0.05$ vs. control. ${ }^{\dagger} P<0.01$ vs. SGN with Ang II. $\# P<0.01$ vs. SGN+AM (Ang II).

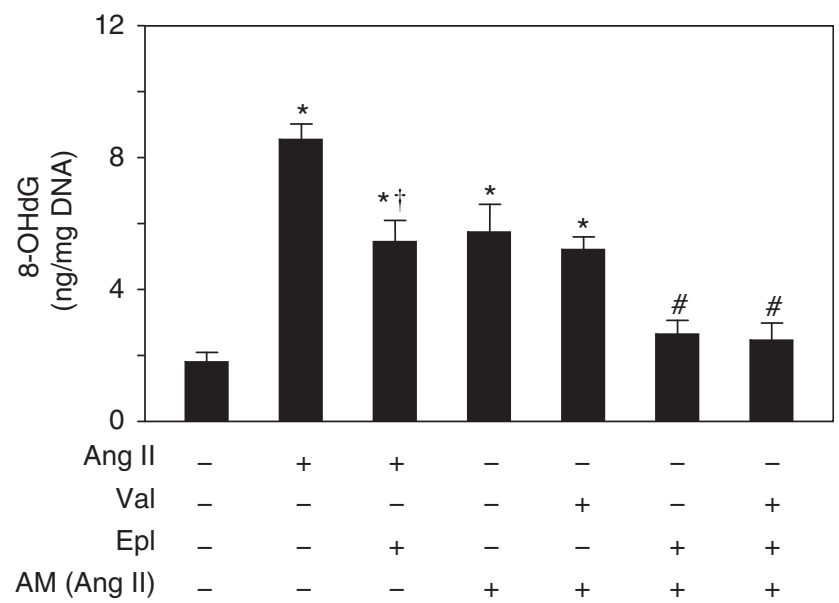

Figure 3 Inhibition of DNA damage by eplerenone in spinal ganglion neurons (SGNs) induced by culture medium from angiotensin (Ang) IItreated astrocytes. Subconfluent and quiescent SGNs were stimulated with or without Ang II in the presence or absence of eplerenone or Ang II-treated astrocyte culture medium in the presence or absence of eplerenone or valsartan. SGNs were stimulated for 3 days and then subjected to 8-hydroxy2 '-deoxyguanosine (8-OHdG) quantification. Values are expressed as mean \pm s.e.m. $(n=4)$. Epl, eplerenone $(10 \mu \mathrm{M})$. AM (Ang II), Ang II-treated astrocyte medium. ${ }^{*} P<0.05$ vs. control. ${ }^{\dagger} P<0.01$ vs. SGN with Ang II. ${ }^{\#} P<0.01$ vs. SGN+AM (Ang II).

Ang II, after which the Aldo concentration in the Ang II-treated astrocyte-conditioned medium was measured. With Ang II stimulation, Aldo concentration in the astrocyte-conditioned medium increased in a time-dependent manner. However, Aldo secretion was 
not observed in the astrocyte-conditioned medium without Ang II stimulation (Figure 4a). We also examined the Aldo concentration in the medium from Ang II-stimulated SGNs. The stimulation methods for SGNs were identical to those for astrocytes. The Aldo concentration in the SGN-conditioned medium increased in a time-dependent manner after Ang II addition (Figure 4b). SGNs were then stimulated with Aldo ( $1 \mathrm{~nm})$ alone for 1,3 and 5 days, and DNA damage was analyzed to determine the possible involvement of Aldo in inducing DNA damage in SGNs. We observed that Aldo stimulation significantly enhanced the 8 -OHdG levels in SGNs in a time-dependent manner starting at 3 days of treatment (Figure $4 b$ ).

\section{Ang II or Aldo stimulation induces cell death in SGNs}

To detect whether Ang II- or Aldo-induced DNA damage in SGNs leads to cell death, we treated SGNs with Ang II or Aldo for 3 days and performed flow cytometric analysis. Figure $5 \mathrm{a}$ shows the dot-plot graph of SGN cell viability; dead cells are localized at the top of the plot. We also quantified the mean percentage of dead SGNs, as shown in Figure 5b. Ang II or Aldo stimulation markedly increased the percentage of dead cells in SGNs to 53.3 or $38.5 \%$ of the total cells, respectively (Figure $5 \mathrm{~b}$ ).

\section{DISCUSSION}

The cellular and molecular mechanisms involved in neuronal degeneration that contribute to neurodegenerative diseases are multi-factor-
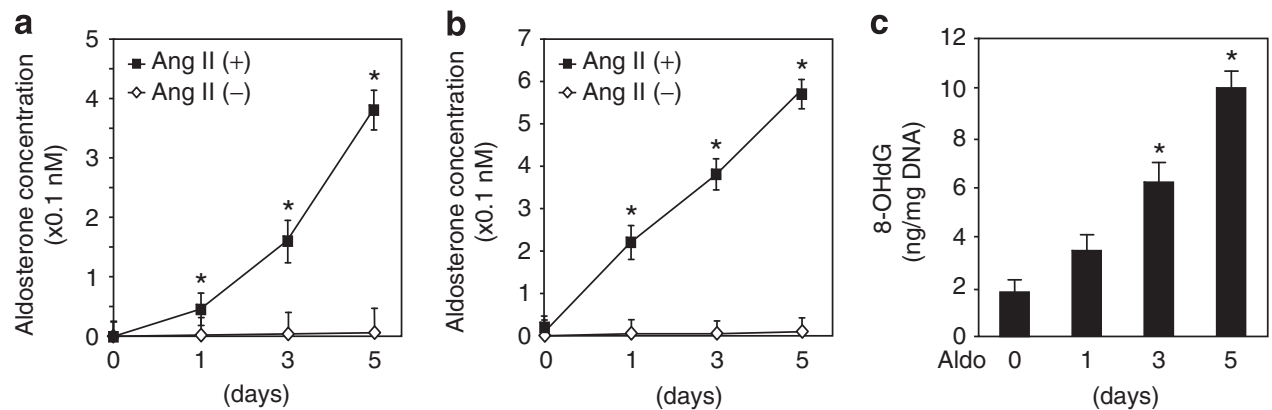

Figure 4 Induction of DNA damage in spinal ganglion neurons (SGNs) by Aldo secreted by Ang II-treated astrocytes. (a) Aldo concentration in the conditioned medium of Ang II-stimulated astrocytes. Subconfluent and quiescent astrocytes were stimulated with or without Ang II for the indicated times. (b) Aldo concentration in the medium of Ang II-stimulated SGNs. Subconfluent and quiescent SGNs were stimulated with or without Ang II for the indicated times. Aldo concentration in the conditioned medium was measured as described in Methods section. Values are expressed as mean \pm s.e.m. ( $n=3$ ). Ang II, angiotensin II (100 nm). ${ }^{*} P<0.05$ vs. Ang II (-) at 1,3 and 5 days, respectively. (c) Aldo stimulation increased DNA damage in SGNs. Subconfluent and quiescent SGNs were stimulated with or without Aldo for the indicated times. Values are expressed as mean \pm s.e.m. ( $n=4)$. Aldo, aldosterone ( $1 \mathrm{~nm}$ ). $* P<0.05$ vs. Aldo 0 day.
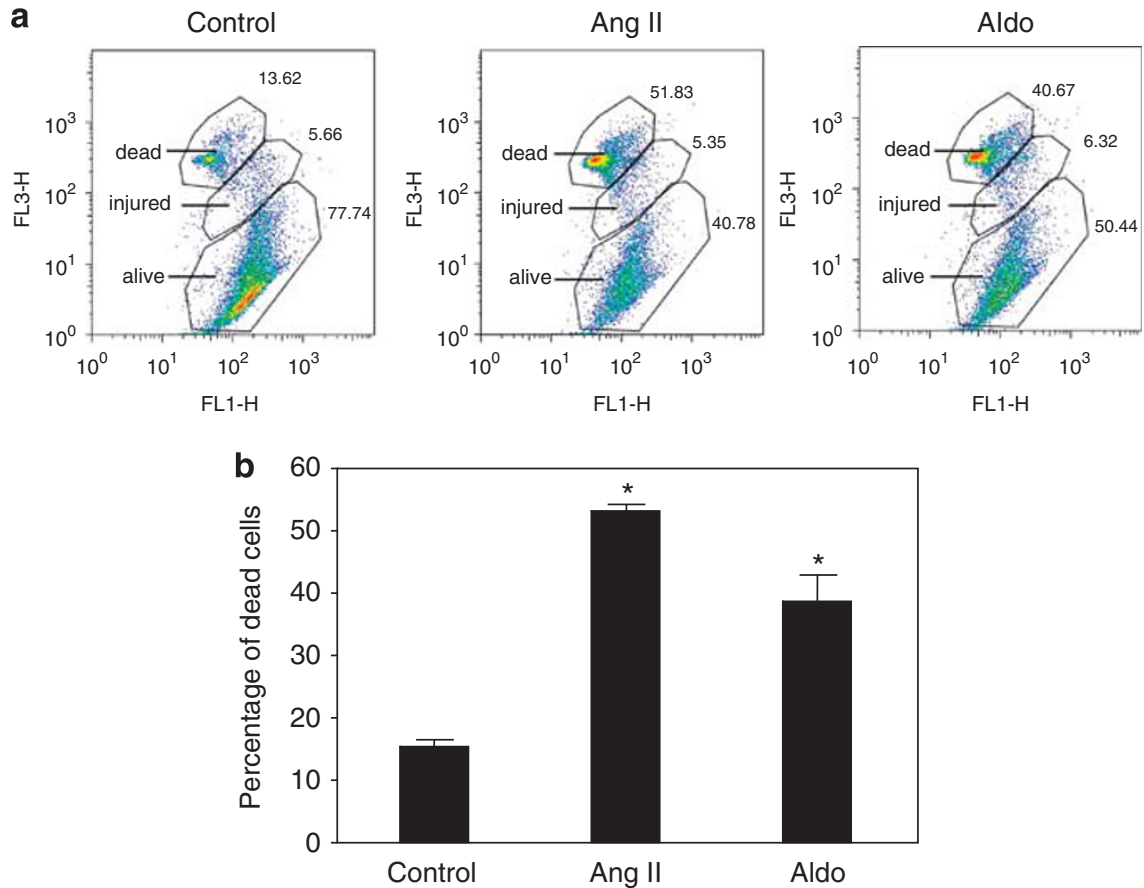

Figure 5 Ang II or Aldo stimulation increases dead cells in spinal ganglion neurons (SGNs). Subconfluent and quiescent SGNs were stimulated with or without Ang II or Aldo for 3 days, after which SGN death was examined by flow cytometric analysis by staining with thiazole orange (TO) and propidium iodide (PI), as described in Methods section. Flow cytometric data were plotted as the cell viability as a function of fluorescence intensity, as shown in (a). (b) Quantification of the mean percentage of dead cells. Values are expressed as mean \pm s.e.m. ( $n=3$ ). Ang II, angiotensin II (100 nм); Aldo, aldosterone (1 nм). ${ }^{*} P<0.01$ vs. control. 
ial and complex. There is evidence implicating oxidative stress as a central mechanism by which neuronal damage occurs. ${ }^{25}$ There is also new evidence indicating that astrocytes expressing a mutated form of SOD1 may contribute to neuronal degeneration. ${ }^{4}$ The mutated SOD1 has been suggested to induce the high production of reactive oxygen species and subsequent DNA damage in neurons. ${ }^{5}$ Therefore, many proposed mechanisms of neuronal degeneration suggest that oxidative stress, together with astrocytes, has an essential role in the pathogenesis of neurodegenerative diseases. However, the link between astrocytes and oxidative stress in causing neuronal damage remains to be elucidated. In this study, we demonstrated that Ang II and Aldo promote neuronal damage and death in SGNs through oxidative stress via the involvement of astrocytes.

Increasing evidence has revealed the important role of local RAAS in CNS disorders. ${ }^{8,9}$ Inhibitors of RAAS, which are widely used as antihypertensive drugs, may have potential beneficial and therapeutic effects on CNS disorders. We and others have shown that treatment with $\mathrm{AT}_{1}$ receptor blockers can reduce the ischemic area in the brain and improve cognitive function in various animal models. ${ }^{26,27} \mathrm{We}$ have also reported that an MR blocker, eplerenone, attenuates stroke size in mice subjected to ischemic brain damage. ${ }^{28}$ Moreover, recent evidence has suggested that $\mathrm{AT}_{1}$ receptor blockers may reduce the incidence of neurodegenerative diseases. Iwasaki et al. ${ }^{11}$ demonstrated that an $\mathrm{AT}_{1}$ receptor blocker, olmesartan, could reduce neuronal death in the spinal cord of the ALS rat in vivo and in vitro. Wang et al. ${ }^{29}$ also reported that another $\mathrm{AT}_{1}$ receptor blocker, valsartan, could protect against brain $\beta$-amyloid-related memory deficit in the $\mathrm{Tg} 2576$ mouse Alzheimer disease model. These findings implicate the $\mathrm{AT}_{1}$ receptor and MR activation as critical mediators in the pathogenesis of CNS disorders, such as neurodegenerative diseases because of neuronal degeneration. Therefore, to further understand the mechanisms involved in neuronal degeneration, the contributory functions of Ang II and Aldo in neuronal degeneration via the $\mathrm{AT}_{1}$ receptor and MR activation have to be elucidated. We demonstrated in this study that Ang II stimulation promotes SGN damage and death starting at 3 days of treatment, as determined by the quantification of the levels of 8-OHdG, a marker of oxidative DNA damage. An $\mathrm{AT}_{1}$ receptor blocker, valsartan, significantly inhibited this Ang II-induced SGN damage. Thus, Ang II might have a role in inducing the SGN damage and death that contributes to the pathogenesis of CNS disorders.

Neuron-astrocyte interaction is involved in the physiological functions of neurons. ${ }^{30}$ However, the contribution of astrocytes to neuronal degeneration has only recently been shown.,4 Therefore, we introduced an SGN and astrocyte co-culture model to verify the hypothesis that astrocytes are involved in SGN damage induced by Ang II stimulation. We demonstrated that Ang II-mediated SGN damage increased in the presence of astrocytes when SGNs were co-cultured with Ang II-stimulated astrocytes. However, co-culture with astrocytes without Ang II stimulation did not induce SGN damage. Thus, it is possible that astrocytes might be involved in Ang II-induced SGN damage. Ang II is well known as a critical mediator of oxidative stress, the increased level of which contributes to the CNS effects of Ang II. ${ }^{20}$ Therefore, we hypothesized that oxidative stress might be tightly linked with Ang II and astrocytes to promote SGN DNA damage under pathophysiological conditions. Our findings also support the importance of neuron-astrocyte interaction in the progression of neuronal degeneration. However, examination of astrocyte reactivity following SGN damage, such as the proliferation of astrocytes, would contribute to further understanding astrocytes as one of the targets for neuroprotection.
The neuronal toxicity of astrocytes expressing SOD1 appears to be partly due to a soluble factor, because the conditioned medium from astrocytes expressing SOD1 is toxic to neurons. ${ }^{4}$ Nerve growth factor and pro-apoptotic mediators have been proposed as the toxic factors. ${ }^{31}$ We examined which toxic factors secreted from astrocytes under Ang II stimulation could cause SGN damage. Recent studies have suggested a reciprocal interaction between Ang II and Aldo in mediating hypertension and cardiovascular disease. Aldo secreted by Ang II stimulation in vascular smooth muscle cells contributes to Ang II-mediated proliferation and senescence. ${ }^{14,32}$ Therefore, we studied whether Aldo was also synthesized and secreted by Ang II-stimulated astrocytes and participated in the progression of SGN damage by culturing SGNs in astrocyte-conditioned medium. Consistent with the above observations, the addition of the culture medium from Ang IItreated astrocytes to SGNs for 3 days stimulated SGN damage and enhanced Ang II-induced damage. Valsartan inhibited the damageinducing effect of Ang II in the conditioned medium from astrocytes. Therefore, it is possible that $\mathrm{AT}_{1}$ receptor signaling could contribute to the formation of toxic factors in astrocytes, thereby resulting in SGN damage in response to Ang II. To examine the possibility that Aldo could act through MR to induce SGN damage, we added valsartan and the MR antagonist eplerenone to SGNs when they were stimulated with the culture medium from Ang II-treated astrocytes. Interestingly, eplerenone strongly inhibited SGN damage induced by the culture medium from Ang II-treated astrocytes, whereas valsartan did not alter DNA damage. Indeed, we also observed that the Aldo concentration was increased in the conditioned medium from Ang IIstimulated astrocytes and SGNs, and that Aldo stimulation also enhanced SGN damage and death starting at 3 days of treatment. Therefore, Aldo, as one of the toxic factors secreted from Ang IIstimulated astrocytes, might further result in the formation of toxic factors in SGNs and contribute to Ang II-mediated SGN damage and death via MR activation. Our findings are consistent with the previous observations that Ang II via $\mathrm{AT}_{1}$ receptor stimulation could induce Aldo release in astrocytes to participate in various pathophysiologic processes, such as hypoxia-associated neurodegeneration. ${ }^{33}$ As Aldo also has a direct role in oxidative stress, ${ }^{21}$ it seems that oxidative stress may also be related to the neuron-damaging effect of Aldo. More detailed examination of the toxic factors that directly cause SGN damage and the signaling pathways involved in Ang II- and Aldoinduced SGN damage with the involvement of astrocytes could contribute to further elucidation of the pathogenesis of CNS disorders.

Recent studies, including ones by our group, have demonstrated that $\mathrm{AT}_{1}$ receptor or Aldo blockers have beneficial and possibly therapeutic effects on CNS disorders. We reported that the $\mathrm{AT}_{1}$ receptor blocker telmisartan prevented ischemic brain damage in diabetic mice, and that telmisartan also has a preventive effect on cognitive impairment in an Alzheimer mouse model, partly as a result of activation of peroxisome proliferator-activated receptor- $\gamma{ }^{34,35}$ These findings suggest that $\mathrm{AT}_{1}$ receptor blockers could exert protective effects on the CNS not only directly through an $\mathrm{AT}_{1}$ receptor or MR blockade, but also via peroxisome proliferator-activated receptor- $\gamma$ activation. These issues should also be clarified in future in vitro studies.

Collectively, the present work shows that Ang II promotes neuronal damage and death in SGNs. In addition, Aldo that is secreted from astrocytes in response to Ang II contributes to Ang II-mediated neuronal damage. Our study identifies potential roles of Ang II and Aldo in inducing neuronal damage through oxidative stress with the involvement of astrocytes. Our findings may provide a novel cellular mechanism of neuronal damage and contribute new insight into the 
possibility that inhibiting Ang II and Aldo with antihypertensive drugs that target the functional integrity of astrocytes could constitute a superior strategy for the treatment of CNS disorders such as neurodegenerative diseases.

\section{ACKNOWLEDGEMENTS}

This work was supported by grants from the Ministry of Education, Science, Sports and Culture of Japan and the Novartis Foundation for Gerontological Research. We thank Dr Kenji Kameda, Integrated Center for Sciences, Ehime University, for technical support of the flow cytometric analysis.

1 Kazantsev AG. Cellular pathways leading to neuronal dysfunction and degeneration. Drug News Perspect 2007; 20: 501-509.

2 Seifert G, Schilling K, Steinhauser C. Astrocyte dysfunction in neurological disorders: molecular perspective. Nat Rev Neurosci 2006; 7: 194-206.

3 Fonseca MI, Kawas $\mathrm{CH}$, Troncoso JC, Tenner AJ. Neuronal localization of $\mathrm{Clq}$ in preclinical Alzheimer's disease. Neurobiol Dis 2004; 15: 40-46.

4 Nagai M, Re DB, Nagata T, Chalazonitis A, Jessell TM, Wichterle H, Przedborski S. Astrocytes expressing ALS-linked mutated SOD1 release factors selectively toxic to motor neurons. Nat Neurosci 2007; 10: 615-622.

5 Barber SC, Mead RJ, Shaw PJ. Oxidative stress in ALS: a mechanism of neurodegeneration and a therapeutic target. Biochim Biophys Acta 2006; 1762: 1051-1067.

6 Wilkinson BL, Landreth GE. The microglial NADPH oxidase complex as a source of oxidative stress in Alzheimer's disease. J Neuroinflam 2006; 3: 30.

7 Unger T, Jakobsen A, Heroys J, Ralph A, Rees T, Shaw M. Targeting cardiovascular protection: the concept of dual renin-angiotensin system control. Medscape J Med 2008; 10(Suppl): S4

8 Brenner D, Labreuche J, Pico F, Scheltens P, Poirier O, Cambien F, Amarenco P, GENIC Investigators. The renin-angiotensin-aldosterone system in cerebral small vessel disease. J Neurol 2008; 255: 993-1000.

9 Mollsten A, Stegmayr B, Wiklund PG. Genetic polymorphisms in the renin-angiotensin system confer increased risk of stroke independently of blood pressure: a nested casecontrol study. J Hypertens 2008; 26: 1367-1372.

10 Kawajiri M, Mogi M, Higaki N, Tateishi T, Ohyagi Y, Horiuchi M, Miki T, Kira JI. Reduced angiotensin II levels in the cerebrospinal fluid of patients with amyotrophic lateral sclerosis. Acta Neurol Scand 2009; 119: 341-344.

11 Iwasaki Y, Ichikawa Y, Igarashi O, Kinoshita M, Ikeda K. Trophic effect of olmesartan, a novel AT1R antagonist, on spinal motor neurons in vitro and in vivo. Neurol Res 2002; 24: 468-472.

12 Carey RM, Siragy HM. Newly recognized components of the renin-angiotensin system: potential roles in cardiovascular and renal regulation. Endocr Rev 2003; 24: 261-271.

13 Marney AM, Brown NJ. Aldosterone and end-organ damage. Clin Sci (Lond) 2007; 113: 267-278.

14 Min LJ, Mogi M, Li JM, Iwanami J, Iwai M, Horiuchi M. Aldosterone and angiotensin II synergistically induce mitogenic response in vascular smooth muscle cells. Circ Res 2005; 97: 434-442.

15 Virdis A, Neves MF, Amiri F, Viel E, Touyz RM, Schiffrin EL. Spironolactone improves angiotensin-induced vascular changes and oxidative stress. Hypertension 2002; 40: 504-510.

16 Ahmad Z, Milligan CJ, Paton JF, Deuchars J. Angiotensin type 1 receptor immunoreactivity in the thoracic spinal cord. Brain Res 2003; 985: 21-31.

17 Oyamada N, Sone M, Miyashita K, Park K, Taura D, Inuzuka M, Sonoyama T, Tsujimoto $\mathrm{H}$, Fukunaga Y, Tamura N, Itoh H, Nakao K. The role of mineralocorticoid receptor expression in brain remodeling after cerebral ischemia. Endocrinology 2008; 149: 3764-3777.

18 Clark MA, Guillaume G, Pierre-Louis HC. Angiotensin II induces proliferation of cultured rat astrocytes through c-Jun N-terminal kinase. Brain Res Bull 2008; 75: 101-106.

19 Chou YC, Luttge WG, Sumners C. Expression of mineralocorticoid type I and glucocorticoid type II receptors in astrocyte glia as a function of time in culture. Brain Res Dev Brain Res 1991; 61: 55-61.

20 Zimmerman MC, Sharma RV, Davisson RL. Superoxide mediates angiotensin II-induced influx of extracellular calcium in neural cells. Hypertension 2005; 45: 717-723.

21 Zhang ZH, Yu Y, Kang YM, Wei SG, Felder RB. Aldosterone acts centrally to increase brain renin-angiotensin system activity and oxidative stress in normal rats. Am J Physiol Heart Circ Physiol 2008; 294: H1067-H1074.

22 Gushchina S, Leinster V, Wu D, Jasim A, Demestre M, Lopez de Heredia L, Michael GJ, Barker PA, Richardson PM, Magoulas C. Observation on the function of nuclear factor kappa B (NF-kappaB) in the survival of adult primary sensory neurons after nerve injury. Mol Cell Neurosci 2009; 40: 207-216.

23 Sporns O, Edelman GM, Crossin KL. The neural cell adhesion molecule (N-CAM) inhibits proliferation in primary cultures of rat astrocytes. Proc Natl Acad Sci USA 1995; 92: 542-546

24 Martinet W, Knaapen MW, De Meyer GR, Herman AG, Kockx MM. Elevated levels of oxidative DNA damage and DNA repair enzymes in human atherosclerotic plaques. Circulation 2002; 106: 927-932.

25 Emerit J, Edeas M, Bricaire F. Neurodegenerative diseases and oxidative stress. Biomed Pharmacother 2004; 58: 39-46.

26 Ito T, Yamakawa H, Bregonzio C, Terron JA, Falcon-Neri A, Saavedra JM. Protection against ischemia and improvement of cerebral blood flow in genetically hypertensive rats by chronic pretreatment with an angiotensin II AT1 antagonist. Stroke 2002; 33 : 2297-2303.

27 Tsukuda K, Mogi M, Li JM, Iwanami J, Min LJ, Sakata A, Fujita T, Iwai M, Horiuchi M. Amelioration of cognitive impairment in the type-2 diabetic mouse by the angiotensin II type-1 receptor blocker candesartan. Hypertension 2007; 50: 1099-1105.

28 Iwanami J, Mogi M, Okamoto S, Gao XY, Li JM, Min LJ, Ide A, Tsukuda K, Iwai M, Horiuchi M. Pretreatment with eplerenone reduces stroke volume in mouse middle cerebral artery occlusion model. Eur J Pharmacol 2007; 566: 153-159.

29 Wang J, Ho L, Chen L, Zhao Z, Zhao W, Qian X, Humala N, Seror I, Bartholomew S, Rosendorff C, Pasinetti GM. Valsartan lowers brain beta-amyloid protein levels and improves spatial learning in a mouse model of Alzheimer disease. J Clin Invest 2007; 117: 3393-3402.

30 Blackburn D, Sargsyan S, Monk PN, Shaw PJ. Astrocyte function and role in motor neuron disease: a future therapeutic target? Glia 2009; 57: 1251-1264.

31 Ricart K, J Pearson Jr R, Viera L, Cassina P, Kamaid A, Carroll SL, Estevez AG. Interactions between beta-neuregulin and neurotrophins in motor neuron apoptosis. J Neurochem 2006; 97: 222-233.

32 Min LJ, Mogi M, Iwanami J, Li JM, Sakata A, Fujita T, Tsukuda K, Iwai M, Horiuchi M. Cross-talk between aldosterone and angiotensin II in vascular smooth muscle cell senescence. Cardiovasc Res 2007; 76: 506-516.

33 Danielyan L, Lourhmati A, Verleysdonk S, Kabisch D, Proksch B, Thiess U, Umbreen S, Schmidt B, Gleiter $\mathrm{CH}$. Angiotensin receptor type 1 blockade in astroglia decreases hypoxia-induced cell damage and TNF alpha release. Neurochem Res 2007; 32: 1489-1498.

34 Tsukuda K, Mogi M, Iwanami J, Min LJ, Sakata A, Jing F, Iwai M, Horiuchi M. Cognitive deficit in amyloid- $\beta$-injected mice was improved by pretreatment with a low dose of telmisartan partly because of peroxisome proliferators-activated receptor- $\gamma$ activation. Hypertension 2009; 54: 782-787.

35 Iwanami J, Mogi M, Tsukuda K, Min LJ, Sakata A, Jing F, Iwai M, Horiuchi M. Low dose of telmisartan prevents ischemic brain damage with peroxisome proliferators-activated receptor- $\gamma$ activation in diabetic mice. J Hypertens 2010; 28: $1730-1737$ 